\title{
On the generality of the partial reinforcement effect
}

\author{
KENNETH R. BURSTEIN \\ Simon Fraser University, Burnaby, British Columbia, Canada V5A 1S6
}

\begin{abstract}
Although currently viewed as the natural, robust, and pervasive effect of intermittent reinforcement, it is suggested (1) that the partial reinforcement effect (PRE) is dependent upon the presence of constraints on the behavioral repertoire of the organism, (2) that it is not generated by the reinforcement schedule per se, and (3) that similar effects can be obtained without any reinforcement.
\end{abstract}

Partially reinforced subjects are more resistant to extinction than are continuously reinforced subjects. This is a statement of "the partial reinforcement effect" (PRE), an effect generally regarded as reflecting one of the most substantial and most powerful phenomena in the area of learning.

Marx (1969, p. 64) notes that: “. . . the PRE is one of the most robust results in instrumental conditioning. Unlike so many other phenomena in instrumental conditioning in which often a number of variables interact in many and sometimes inexplicable ways, the PRE is obtainable under an extremely wide range of conditions and organisms. There is some question whether it occurs in fish, but at the mammalian level it has something of the status of a behavioral law."

The importance and the inherentness of the PRE is similarly stressed by Deese and Hulse (1967, p. 154): "The effect of patterns of reinforcement upon resistance to extinction is of the greatest theoretical, practical, and adaptive significance. For one thing, we have a key to the understanding of why behavior in natural settings is likely to be so persistent: most behavior is probably not reinforced for all tries, and when reinforcement does come, it tends to come in amounts which vary from try to try. It is fortunate that organisms have evolved to respond the way they appear to do to patterns of reinforcement, since the real world seems to be arranged so that reinforcement is inconsistent, often unpredictable, even capricious."

The intent of this paper is to suggest that the PRE is not the natural consequence of partial reinforcement, that it is not generated by the reinforcement schedule per se, and that similar if not identical effects can be obtained without intermittent reinforcement.

The unanimity of textbooks on learning with respect to the robustness of the PRE attests to the wealth of data supporting the phenomena. We have no questions whatsoever about the validity of the data and of the studies which are presented to support the generality of PRE.

We do suggest, however, that there are commonalities in all of these situations which seem to have been over- looked, and which may have resulted in a misinterpretation with respect to the generality of the PRE.

Let us select a few haphazardly chosen, but typical, illustrations of the PRE: (1) An infant who has been allowed to cry for several hours before being picked up or attended to, continues to cry for hours when he is not attended to. (2) Patrons at a Las Vegas casino persistently play "one-armed bandits" although there are relatively few payoffs. (3) A pigeon (or rat) is placed in an operant conditioning chamber and keypecks (or barpresses) are rewarded on a variable-interval or variableratio schedule. (4) Dating behavior persists although the rewards which are sometimes envisioned seldom materialize. (5) Authors continue to submit articles to publishers although the rate of acceptance is fairly low.

How robust is the effect described in Example 1? If an infant is placed on a variable-ratio (or variableinterval) schedule so that his screams are rewarded, say, on the average, every hour, how long will the PRE last? The continuance of such a schedule may result in periodically consistent screaming for days, or even months. But at the risk of appearing frivolous, what if the schedule was continued ad infinitum?

We suspect that, at the age of 10-12 months, the child would come waddling or walking out of his room, unless he was confined in a crib. If this were the case, we suspect that the periodic screaming (PRE) would persist until the child could overcome his confinement by climbing out of the crib.

The critical point is that this particular "robust" PRE effect, rather than reflecting a natural, pervasive behavior typical of all humans, can be obtained only with confined, nonlocomoting infants. It would be impossible to obtain this PRE with normal 4-year-olds, teen-agers, or adults presented with identical schedules of reinforcement in identical situations.

This PRE effect could be reinvoked in postinfantile human subjects by reinstating the conditions of confinement under which the infant exhibits the effect. Thus, a hospitalized adult in traction might exhibit a similar PRE effect to that described in Example 1, if he were in distress and had no other available means of com- 
munication with the nurse or anyone else who might provide aid. An adult would hardly resort to continued cries for help unless those cries were his only means of summoning aid.

It should be clear, therefore, that at least in this particular illustration of the PRE the effect is dependent upon the availability of alternative behaviors. This PRE is not obtained with normal adults because their behavior repertoires include locomotion to determine why their cries have gone unheeded, exploration of their environment in order to alleviate their own distress, etc. In a partially confining situation, e.g., in traction in a hospital, he can ring for a nurse, and if the ringing is partially reinforced, the PRE may be obtained. In the absence of any reinforcement for bell ringing, the human adult may "regress" to periodic (PRE) screaming.

This illustration is not facetiously intended. We suggest that, in every case of the PRE, the subject is similarly restricted in terms of behavioral alternatives, and that without this restriction the PRE would not be obtained.

One-armed bandit players have no other possibility of obtaining immediate large rewards for relatively small investments. The pigeon or rat in an operant chamber has no other fairly immediate means of obtaining foodand it has previously been severely deprived of food. Whatever the rewards of dating are, there is no other socially acceptable behavioral alternative for obtaining them. "Publish or perish" is a slogan which reflects the same lack of alternative behaviors for "success."

The essence of psychological research is such that virtually any subject, in any experiment, has had his, or its, behavioral repertoire restricted by Physical or instructional manipulation. If the PRE was a natural and general phenomenon generated by the reinforcement schedule per se, all vending machines would be programmed like one-armed bandits. It is a lack of recogni- tion of the restrictive elements necessary to obtain the PRE or high rates of response with partial reinforcement that once led a graduate school colleague of ours to suggest that factory workers should be paid on a partial reinforcement schedule. Such an "acquisition" schedule would result in low, rather than high, levels of performance, for, under normal circumstances, there are alternative sources of reinforcement. If such alternative sources of employment were eliminated, the PRE would be obtained as routinely as it is now reported in the psychological literature.

Perhaps it is even more important to recognize that the high rates of response obtained during both partially reinforced acquisition and in the PRE are primarily dependent upon the "imprisonment" of the subject, be it physical, psychological, or instructional, rather than the schedule of reinforcement employed.

Under conditions where the behavioral repertoire of the subject is restricted, performances comparable to those reported with the PRE can be, and are, obtained without any reinforcement whatsoever. Dating behavior can be persistent without any reinforcement. Some writers continue to submit articles to journals although none have ever been accepted. A chronic smoker will continue to "play" the only cigarette machine to which he has access. People continually enter beauty, chess, cooking contests, etc., without ever coming close to victory. Indeed, the "zero-reinforcement effect" may be as robust and natural as the PRE is thought to be.

\section{REFERENCES}

DeEse, J.. \& Hulse, S. H. The psychology of learning. New York: McGraw Hill, 1967.

MarX. M. H. Learning: Processes. New York: Macmillan, 1969.

(Received for publication February 19, 1976.) 